\title{
Butyrate Regulates the Expression of Pathogen-Triggered IL-8 in Intestinal Epithelia
}

\author{
MEIQIAN WENG, W. ALLAN WALKER, AND IAN R. SANDERSON
}

\begin{abstract}
Department of Pediatrics [M.W., W.A.W.], Division of Pediatric Gastroenterology and Nutrition, Massachusetts General Hospital and Harvard Medical School, Charlestown, Massachusetts 02129; Research Centre for Gastroenterology [I.R.S.], Barts and The London, Queen Mary's School of Medicine and Dentistry, London E1 2AT, United Kingdom
\end{abstract}

\begin{abstract}
Inflammatory bowel disease (IBD) is characterized by an exaggerated immune response that involves pro-inflammatory cytokines including IL-8. Production of these pro-inflammatory cytokines is triggered by pathogen-associated molecular patterns (PAMP). Butyrate, a product of bacterial fermentation of carbohydrates, has been reported to modulate inflammation in IBD, possibly by regulating production of pro-inflammatory cytokines. However, this effect of butyrate is controversial. In this study, we used Pam3CSK4 (Pam3CysSerLys4), the acylated $\mathrm{NH}_{2}$-terminus of the bacterial lipoprotein (a PAMP), to mimic in vivo infection of pathogens. Butyrate transiently down-regulated expression of IL-8 stimulated by Pam3CSK4. Treatment of cells with butyrate before Pam3CSK4, however, enhanced production of IL-8. Furthermore, butyrate induced expression of A20, a negative regulator of the nuclear factor- $\kappa \mathrm{B}$ pathway. Over-expression of A20 inhibited Pam3CSK4-triggered IL-8 expression. Our data suggest that the inflammatory modulation of butyrate in IBD is mediated by A20 and a short pulse rather than continuous administration of butyrate may provide a protective effect on IBD. (Pediatr Res 62: 542-546, 2007)
\end{abstract}

$\mathrm{I}^{\mathrm{n}}$ nflammatory bowel disease (IBD) epitomizes chronic inflammatory intestinal disorders that comprise two similar but different disorders, ulcerative cells (UC) and Crohn's disease (CD). The etiology of IBD, however, is unclear but believed to involve an excessive immune response. Epithelial cells that line the inner layer of the intestine tract supply a barrier for entrance of pathogenic microbes. In addition, epithelial cells actively contribute to pathogenesis of IBD by secreting chemokines (1). Given that epithelial cells are positioned to encounter pathogens before immune cells including neutrophils, the production of chemokines from epithelial cells in response to infection of pathogens is the key step for the host immune system to embark on the effective clearance of pathogens. This also points to the important role of epithelial cells in initiating IBD.

Received March 19, 2007; accepted June 4, 2007.

Correspondence: W. Allan Walker, M.D., Department of Pediatrics, Developmental Gastroenterology Laboratory, Division of Pediatric Gastroenterology and Nutrition, MA General Hospital for Children and Harvard Medical School, 114 16th Street (1143503), Charlestown, MA 02129; e-mail: wwalker@partners.org

This work was supported by the Fogarty International Center for Child Health and Human Development Training Grant (D43-TWO 01265). I.S. was on paid sabbatical leave from Queen Mary, University of London, during the course of these studies. The tsFHI cells used in this study were a gift from Dr. Andrea Quaroni (Department of Biomedical Sciences, Cornell University, Ithaca NY). An A20 plasmid used in this study was obtained from Dr. Claudius Vincenz's lab (HHMI, Ann Arbor, MI).
Butyrate, a short-chain fatty acid, is endogenously produced by bacterial fermentation of carbohydrates. In consideration of its modulation of inflammation, butyrate has been used as a supplementary treatment of UC. This modulation of butyrate is considered to arise at least in part from regulating production of cytokines and/or chemokines. However, the effect of butyrate on the expression of cytokines and/or chemokines from intestinal epithelial cells is conflicting. Different studies reported that butyrate could both up- and down-regulate production of IL-8 (2-6). The up-regulatory effect may result from its chromatin remodeling action by inhibiting histone deacetylase (2). However, no satisfactory model is available to interpret its down-regulation of IL-8 expression.

In this study, by monitoring IL-8 production, we recapitulated the response of epithelial cells to Pam3CSK4 to mimic the in vivo response of the intestinal epithelial cells to infection of pathogenic and/or commensal microbes. Based on this in vitro inflammation model, we explored the role of butyrate in the protection against the inflammation in gut. We found that butyrate transiently down-regulated production of Pam3CSK4-stimulated IL-8 from both epithelial cell lines and human intestine explants by inducing expression of A20, a negative regulator of the nuclear factor (NF)- $\kappa$ B pathway.

\section{MATERIALS AND METHODS}

Cell culture. The human intestinal cell lines, Caco-2 and SW480 (20-30 passages, ATCC, Manassas, VA), were grown in Dulbecco's modified Eagle's medium (DMEM) supplemented with 10\% fetal bovine serum (FBS), 2 $\mathrm{mM}$ glutamine, $50 \mathrm{U} / \mathrm{L}$ penicillin, and $50 \mu \mathrm{g} / \mathrm{L}$ streptomycin, $10 \mathrm{mM}$ HEPES buffer solution, and $1 \%$ nonessential amino acid. All cell culture reagents were obtained from Invitrogen (Carlsbad, CA). Temperature sensitive fetal human intestinal epithelial cells (tsFHI) cell lines were a kind gift from Dr. Andrea Quaroni. tsFHI cells were grown at $32^{\circ} \mathrm{C}$ in growth medium consisting of OptiMEM I supplemented with $4 \%$ heat-inactivated FBS, 10 ng/mL EGF, 2 mM glutamine, 2 mM L-alanyl-glutamine (GlutaMAX I), $10 \mathrm{mM}$ HEPES, $50 \mathrm{U} / \mathrm{mL}$ penicillin, and $50 \mu \mathrm{g} / \mathrm{mL}$ streptomycin. When confluent, cells were transferred to $37^{\circ} \mathrm{C}$ and medium consisting of DMEM supplemented with 7\% heat-inactivated FBS, 2 mM glutamine, 2 mM GlutaMAX I, $10 \mathrm{mM}$ HEPES, $50 \mathrm{U} / \mathrm{mL}$ penicillin, and $50 \mu \mathrm{g} / \mathrm{mL}$ streptomycin. Cells were then used $7 \mathrm{~d}$ later. Cells were stimulated with the synthetic bacterial lipoprotein (BLP) substitute, Pam3CSK4, a recog-

Abbreviations: BLP, bacterial lipoprotein; CD, Crohn's disease; IBD, inflammatory bowel disease; IEC, intestinal epithelial cell; PAMP, pathogenassociated molecular patterns; TLR, Toll-like receptor; tsFHI, temperature sensitive fetal human intestinal epithelial cells; TMB, tetra methyl benzidine; UC, ulcerative colitis 
nized toll-like receptor (TLR)2 agonist at varying doses for different time periods.

ELISA. IL-8 was measured in intestinal epithelial cell (IEC) cell cultured supernatants by ELISA in duplicates as described previously (7). Ninety-sixwell microtiter plates (Immulon 2HB) were coated overnight with anti-human IL-8 (R \& D systems, Minneapolis, MN), and then incubated with rabbit anti-human IL-8 (Endogen, Rockford, IL), horseradish peroxidase (HRP)conjugated goat anti-rabbit IgG (Biosource, Camarillo, CA), and tetra methyl benzidine (TMB) peroxidase substrate (BioFX Labs, Owings Mills, MD). Colorimetric results were read on a plate reader at a wavelength of measured at $450 \mathrm{~nm}$. The variation between duplicates was less than $2 \%$. IL-8 values were normalized against total cellular protein as determined with the DC Protein assay (Bio-Rad, Hercules, CA) following the manufacturer's instructions. Cells were removed by ultrasonic disruption, as previously described in IEC cell lysate buffer [100 mM HEPES (pH 7.4), $150 \mathrm{mM} \mathrm{NaCl,} 2 \mathrm{mM}$ $\mathrm{CaCl}_{2}, 2 \mathrm{mM} \mathrm{MgCl} \mathrm{Mg}_{2}, 0.2 \mathrm{mM}$ EDTA, $0.5 \mathrm{mM}$ PMSF, $50 \mathrm{mM} \mathrm{NaF}, 1 \mathrm{mM}$ $\mathrm{NaVO}_{4}, 0.1 \%$ triton X-100].

Electroporation of siRNA. Small interfering RNA (siRNA) were purchased from Qiagen (Valencia, CA). The sense strand sequence of the RNA duplexes designed against human A20 was $5^{\prime}$-r(GGU AGA UGA UUA CUU UGA A) dTdT-3'. The noncomplementary siRNA 5'-UUC UCC GAA CGU GUC ACG UdT dT-3' was used as the negative control siRNA. The pmaxGFP ${ }^{\mathrm{TM}}$, which encodes the green fluorescent protein (GFP) from Copepod Potellina P, was used as the positive control to assess transfection efficiency. The siRNA was transfected into cells by electroporation using Cell Line Nucleofector Kit T (Amaxa Inc., Gaithsburg, MD). One microgram siRNA of A20 was transfected into Caco-2 cells. $48 \mathrm{~h}$ after siRNA treatment, Caco-2 cells were stimulated with Pam3CSK4 50 $\mu \mathrm{g} / \mathrm{mL}$ (Invitrogen), for another $18 \mathrm{~h}$, after which cells were harvested with lysate buffer and culture medium was collected for ELISA assay.

Transfection of Caco-2 Cells with A20 cDNA. A20 plasmid was cloned by $\mathrm{XbaI}$ into the $\mathrm{pCB} 6 / \mathrm{pJDM} \cdot 464$ vector. It was the generous of gift from Dr. Claudius Vincenz. It was transfected into cells by electroporation, as described above.

Protein transfer (Western) blot analysis cells were exposed to Pam3CSK4, and/or butyrate for varying times as indicated in the text. Cells were then washed with PBS and lysed in buffer containing $1 \%$ Nonidet P-40, $20 \mathrm{mM}$ Tris- $\mathrm{HCl}$ (pH 7.4), $150 \mathrm{mM} \mathrm{NaCl}, 2$ mM EDTA, 2 mM EGTA, 2 mM PMSF, phosphatase inhibitors, and protease inhibitor cocktail tablets. Lysates were homogenized, and protein contents were determined with the DC Protein assay. For Western blotting, $20 \mu \mathrm{g}$ of protein from each sample was subjected to SDS-PAGE on a 4-20\% gradient Tris-Glycine Gel (BioRad) under reducing conditions. Proteins were then transferred onto a PVDF or nitrocellulose membrane (Bio-Rad) and blocked with TBST (Tris-buffered saline, Tween 20) (with $5 \%$ dry milk) overnight at $4^{\circ} \mathrm{C}$, followed by incubation for $1 \mathrm{~h}$ at room temperature with primary antibody diluted in blocking buffer at 1:1000 for each of the following antibodies: A20 (AXXORA LAA), pI $\kappa$ B alpha (Cell Signaling Technology, Beverly, MA). Membranes were then washed with TBST for $30 \mathrm{~min}$, followed by incubation for $1 \mathrm{~h}$ at room temperature with second antibody (conjugated with HRP) diluted in blocking buffer at 1:5000 (Bio-Rad).

Human intestine organ culture. To examine the expression of A20 in human intestine organ culture, fetal intestine (15-24 wk) was used. The intestine was obtained from the Harvard Nutrition Research Center tissue core (Dr. Nanda Nanthakumar, Director). The use of this material had full permission from the institutional review board. It was washed with fresh serum-free DMEM supplemented with $2 \mathrm{mM}$ glutamine, $50 \mathrm{U} / \mathrm{L}$ penicillin, and $50 \mu \mathrm{g} / \mathrm{L}$ streptomycin, $10 \mathrm{mM}$ HEPES buffer solution, and $1 \%$ nonessential amino acid with transferrin $(5 \mathrm{mg} / \mathrm{L})$ and selenous acid $(5 \mu \mathrm{g} / \mathrm{L})$. It was then divided into small pieces, placed into the 48 wells, and maintained in fresh culture medium at $37^{\circ} \mathrm{C}$ in an environment of $95 \% \mathrm{O}_{2} / 5 \% \mathrm{CO}_{2}$ for $6 \mathrm{~h}$.

Statistics. Results are presented as mean values \pm SD. Statistical significance was determined using $t$ test. Unless otherwise stated, $n=3 ; p<0.05$ was considered statistically significant.

\section{RESULTS}

Elevated IL-8 secretion by Pam3CSK4-stimulated Caco-2 cells. Production of chemokines, such as IL-8, by epithelial cells in response to microbes attracts neutrophils to infected loci and the recruited neutrophils secret more cytokines and chemokines for recruiting immune cells and more neutrophils to initiate the specific immune response. Chemokine production from epithelial cells, therefore, might be a central step in the pathogenesis of IBD. Butyrate can regulate the production of such chemokines from epithelial cells, thereby in part addressing its modulating effect on inflammation.

To further study the effect of butyrate on modulating inflammation, we first established an in vitro inflammatory model using Pam3CSK4, a TLR2 ligand (8), to mimic the BLP, a pathogen-associated molecular pattern (PAMP), whose receptor is TLR2. Upon treatment with Pam3CSK, phosphorylated $\mathrm{I} \kappa \mathrm{B} \alpha$ gradually increased in Caco-2 cells (Fig. $1 A$ ), indicating the activation of the $\mathrm{NF} \kappa \mathrm{B}$ pathway (9). Consequently, in the presence of Pam3CSK4, Caco-2 cells secreted IL-8 into the culture medium to a significantly elevated level (Fig. 1B). These data suggest that in vitro administration of Pam3CSK4 to epithelial cells can mimic the in vivo response of the intestinal epithelia to infection of pathogens.

Butyrate transiently down-regulates Pam3CSK4-triggered IL-8 in intestinal epithelial cells. Having established the in vitro inflammatory model, we next investigated the effect of butyrate on the production of Pam3CSK4-stimulated IL-8 in Caco- 2 cells. Again, treatment of cells with Pam3CSK4 stimulated the secretion of IL-8 into the culture medium. However, cells treated with PamCSK4 and butyrate significantly inhibited the secretion of IL-8 compared with cells treated with Pam3CSK4 alone (Fig. 2A). The down-regulation of IL-8 is time dependent. Co-treatment of cells for more than $6 \mathrm{~h}$, however, resulted in an increase of IL-8 secretion (Fig. 2A). This transient down-regulation of Pam3CSK4-stimulated IL-8 was also observed in other cell lines (Fig. 2, $B$ and $C$ ). The duration of inhibiting Pam3CSK4-triggered IL-8 secretion varied with the different cell lines. In the immature tsFHI cells (Fig. 2B), the inhibitory effect of butyrate on Pam3CSK4evoked IL-8 secretion lasted longer than that in mature Caco-2 (Fig. 2A) and SW480 (Fig. 2C) cells. Thus, the differences between Caco-2/SW480 and tsFHI may result from the mat-

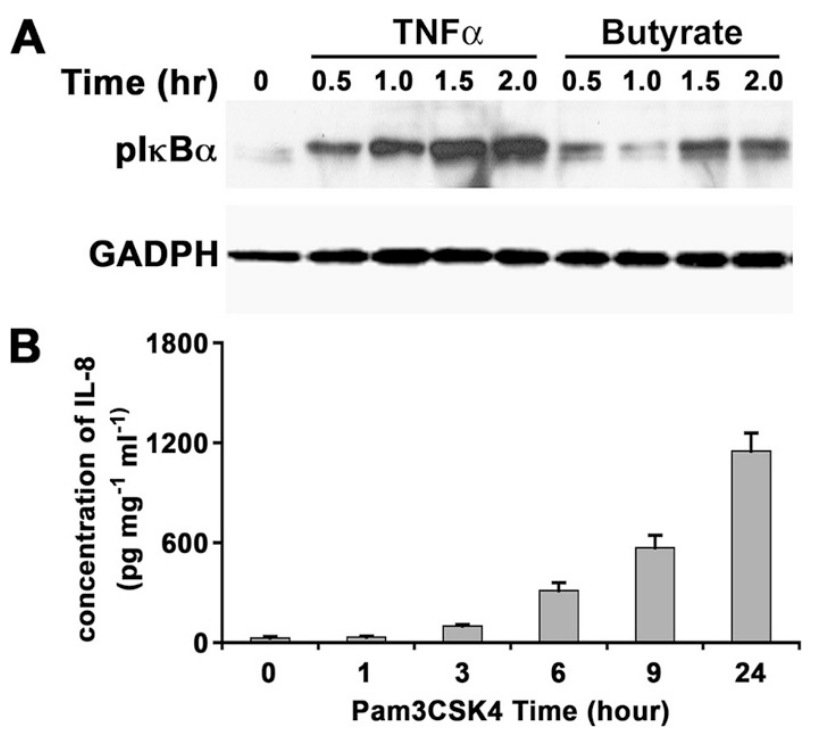

Figure 1. Pam3CSK4-evoked IL-8 secretion by Caco-2 cells mimics the in vivo response of intestinal epithelia to pathogens. Caco- 2 cells were stimulated with Pam3CSK4 at a final concentration of $50 \mu \mathrm{g} / \mathrm{mL}$ for indicated times. Postnuclei supernatants were prepared for detection of $\mathrm{pI} \kappa \mathrm{B} \alpha(A, n=3)$, and culture media were collected for measurement of IL- 8 by $\operatorname{ELISA}(B, n=3)$. Data are represented as mean $\pm \mathrm{SD}$. 

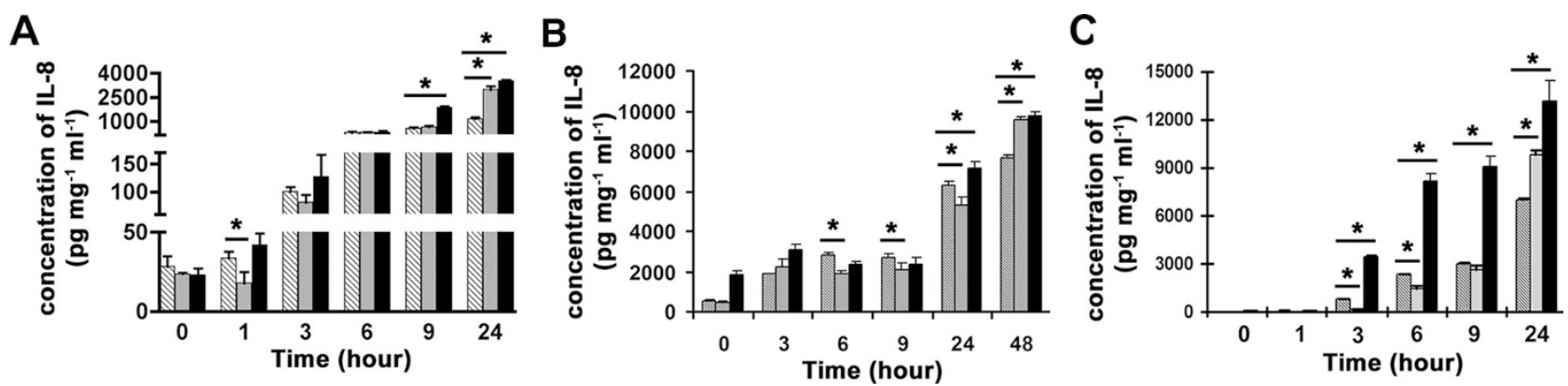

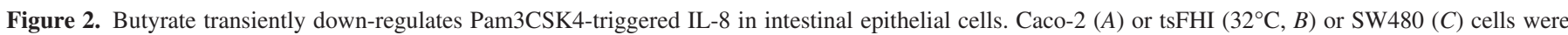

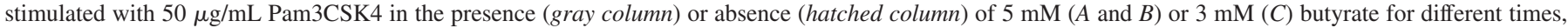

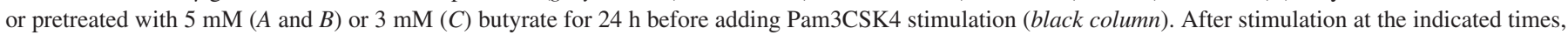
the concentration of IL-8 in culture media was measured by ELISA and plotted. Data are represented as mean \pm SD $(n=3$, $* p<0.05)$.

uration stage of these cell lines. Of note, however, incubation of cells with butyrate before treatment with Pam3CSK4 had no beneficial effect on inhibition of IL-8 production. In contrast, preincubation with butyrate exacerbated Pam3CSK4evoked IL-8 expression (Fig. 2, A-C). In fact, butyrate alone can stimulate expression of IL-8 in epithelial cells, as previously reported. This stimulatory effect is consistent with the action of butyrate on gene transcription by remodeling chromatins through inhibiting histone deacetylation $(2,10)$.

Butyrate functions via A20. Pam3CSK4 stimulated IL-8 via activating the NF- $\kappa$ B pathway (Fig. 1). We therefore examined whether speculated that butyrate regulated A20 through the NF- $\kappa \mathrm{B}$ pathway. To test this possibility, we first determined whether treatment with butyrate could change the level of phosphorylated I $\kappa \mathrm{B} \alpha$. As shown in Figure $3 A$, butyrate had no effect on phosphorylation of $\mathrm{I} \kappa \mathrm{B} \alpha$, implying that butyrate acted through a different mechanism from the NF- $\kappa \mathrm{B}$ pathway.

Recent studies demonstrated that A20 inhibited the activation of transcription factors such as NF- $\kappa \mathrm{B}$ and AP-1, both of which induce IL-8, through the TLR4 pathway $(11,12)$. We therefore asked whether butyrate functions via A20. If this were the case, because A20 is constitutively expressed in epithelial cells (Caco-2) cells at a low level, we expected that butyrate would enhance the expression of A20, and that a gain of A20 function would inhibit Pam3CSK4-evoked IL-8 secretion. As shown in Figure $3 B$, butyrate indeed induced expression of endogenous A20.

Next, we examined the effect of gain of A20 function on Pam3CSK4-triggered IL-8 production in Caco-2 cells (Fig. $3 C$ ). As expected, overexpression of exogenous A20 (gain of A20 function) significantly decreased Pam3CSK4-triggered IL-8 production. The inhibition of Pam3CSK4-triggered IL-8 production by A20 is specific since transfection of empty vector had no effect on Pam3CSK4-triggered IL-8 secretion (Fig. 3D). For further evidence, we established siRNAmediated knockdown of A20. As shown in Figure 3E, transfection of A20-specific siRNA significantly reduced the level of endogenous A20. Treatment of these cells transfected with A20-specific siRNA with Pam3CSK4 led to significant elevation of IL-8 compared with cells transfected with nonspecific siRNA (Fig. 3F). These data suggest that A20 plays an important role in IL-8 expression through the TLR2 pathway activated by Pam3CSK4.
Taken together, butyrate transiently down-regulates Pam3CSK4-triggered IL-8 production by inducing expression of A20, a negative regulator of the NF- $\kappa \mathrm{B}$ pathway.

Butyrate enhances expression of A20 in human intestine explants. Our in vitro data showed that butyrate provides a time-limited protective effect against inflammation initiated by epithelial cells. This effect is achieved by A20-mediated inhibition of the NF- $\kappa \mathrm{B}$ pathway, thereby leading to reduced secretion of chemokine IL-8. We asked whether this phenomenon also occurred in vivo. As we were unable to study this effect of butyrate directly on patients, we took advantage of human intestinal explants. To maximally mimic physiologic conditions, segments of human intestine were cultured ex vivo for $6 \mathrm{~h}$ in the presence or absence of butyrate or Pam3CSK4. Unlike cell lines, Pam3CSK4 did not significantly induce human intestine explants to secret IL-8 on exposure for $6 \mathrm{~h}$ (Fig. 4A), as the explants have a high background production of IL-8 without stimulation. It is possible that the human intestine explants had already been exposed to commensal bacteria harboring PAMP in the uterus. Alternatively, the exposure duration is not long enough to induce the significant production of IL-8 in human explants. However, we were unable to culture human intestine explants for a longer time preventing us from investigating this possibility further. Other possibilities might also exist. For example, the stimulatory effect of Pam3CSK4 is counterbalanced by other factors since explants contain a complex of cells including immune cells. Nevertheless, treatment of human intestine explants with butyrate decreased the secretion of chemokine IL-8 (Fig. 4A). However, this effect will need to be confirmed by investigating patients directly. As shown in Figure $4 B$, butyrate augmented expression of A20 in human intestine explants, suggesting that the effect of butyrate on suppressing IL- 8 in human intestine explants is also dependent on A20.

\section{DISCUSSION}

In this study, we recapitulated the response of epithelial cells or intestine explants to Pam3CSK4 to investigate the contribution of epithelial cells to IBD and explored the possible mechanism for butyrate to modulate inflammation in IBD. We found that butyrate transiently down-regulates 
A

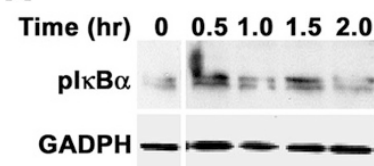

B

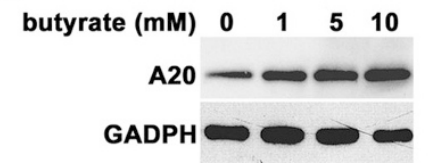

C

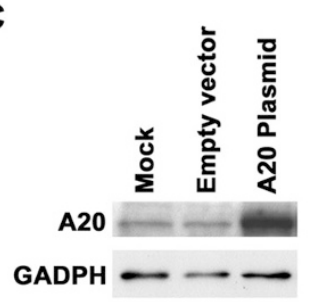

$\mathbf{E}$

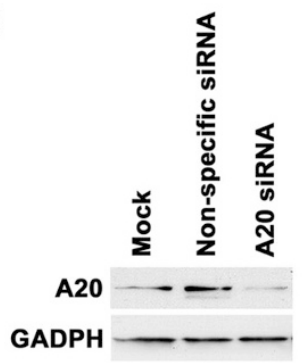

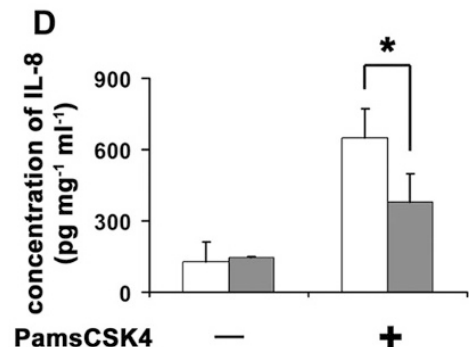

$\mathbf{F}$

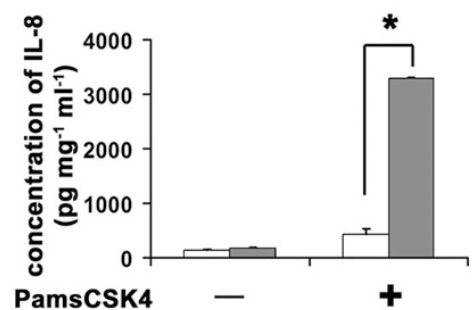

Figure 3. A20 expression is altered by butyrate, and it inhibits IL-8 secretion. (A) Butyrate acts independently of $\mathrm{pI} \kappa \mathrm{B} \alpha$. Caco- 2 cells were incubated with $5 \mathrm{mM}$ butyrate for the indicated times. Postnuclei supernatants were prepared and analyzed by Western blotting with indicated antibodies. The result for $0 \mathrm{~h}$ is from the same experiment as in Figure 1A. (B) Butyrate induces the expression of A20. Caco-2 cells were treated with various concentrations of butyrate for $3 \mathrm{~h}$. Total cell lysates were prepared for immunoblotting with antibody against A20 and normalized by GAPDH. $(C-F)$ A20 gain- and loss-of-function regulate PamsCSK4-stimulated secretion of IL-8 from Caco-2 cells. (C) Expression of exogenous A20. Caco-2 cells were transfected with A20 plasmid or the corresponding empty vector (mock) for $24 \mathrm{~h}$. Postnuclei supernatants were prepared for detection of exogenous A20 expression normalized by GAPDH. (D) Expression of exogenous A20 significantly inhibits Pam3CSK4-triggered IL-8 production. After transfection with A20 plasmid (gray column) or empty vector (white column) for $24 \mathrm{~h}$, Caco-2 cells were stimulated with $50 \mu \mathrm{g} / \mathrm{mL}$ Pam3CSK 4 for $18 \mathrm{~h}$. The concentration of IL-8 in the culture medium was measured by ELISA and plotted $(n=3$, mean $\pm \mathrm{SD}, * p<0.05)$. (E) Knockdown of endogenous A20 in Caco- 2 cells. A20-specific or nonspecific siRNAs were delivered into Caco-2 cells by electroporation as described in "Materials and Methods." After 24 h, postnuclei supernatants were prepared for Western blotting analysis with indicated antibodies. $(F)$ A20 loss-of-function significantly increases Pam3CSK4-triggered IL-8 production, Caco-2 cells were transfected with A20-specific (gray column) or nonspecific siRNAs (white column) for $48 \mathrm{~h}$ and then stimulated by Pam3CSK4 for $18 \mathrm{~h}$. The concentration of IL-8 in the culture medium was measured by ELISA and plotted $(n=3$, mean \pm $\mathrm{SD}, * p<0.05)$

PAMP-stimulated production of chemokine IL-8 dependent on $\mathrm{A} 20$, a negative regulator of the NF- $\kappa \mathrm{B}$ pathway.

Neutrophil transmigration across mucosal epithelia is considered a hallmark of inflammatory conditions in IBD. In this regard, production of chemical factors that usher neutrophils to infectious loci would be the key step in IBD. The intestinal epithelium is the first line of host defense against pathogens, presenting PAMP, such as lipopolysaccharide (LPS), bacterial lipoprotein, peptidoglycan (PGN), flagellin, and CpGcontaining (unmethylated) DNA. Interestingly, epitheliaoriginated IL- 8 has been shown to trigger neutrophil migration

A

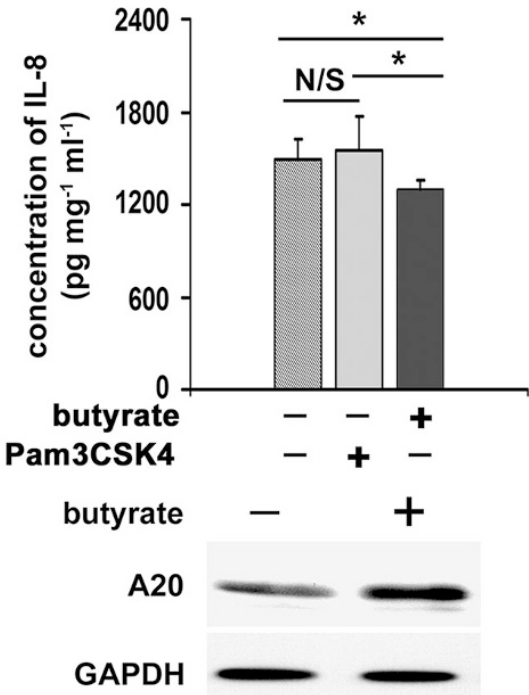

Figure 4. Effect of butyrate on human fetal intestinal explants. (A) Butyrate decreases the expression of IL-8 in short incubations. Human fetal intestine explants were cultured in the presence or absence of $5 \mathrm{mM}$ butyrate or 50 $\mu \mathrm{g} / \mathrm{mL}$ Pam3CSK 4 for $6 \mathrm{~h}$. The culture medium was collected, and the secreted IL- 8 was measured by ELISA and plotted $(n=3$ pieces, mean \pm SD, ${ }^{*} p<0.05 ; \mathrm{N} / \mathrm{S}$, no significance). Shown are data from one patient. Similar results were observed in other two patients. $(B)$ Butyrate enhances expression of A20 in human intestine explants. A segment of human fetal intestine (18 wk) was divided into three parts and cultured in the presence or absence of 5 $\mathrm{mM}$ butyrate for $6 \mathrm{~h}$. Lysates were prepared and the post debris supernatants were analyzed by immunoblotting with antibody against A20, GAPDH was used as the loading control.

in vitro $(13,14)$. Furthermore, the transgenic expression of the IL-8 homolog, macrophage inflammatory protein-2 (MIP-2), in mouse epithelium attracted neutrophils into the lamina propria in vivo (15). These data directly point to, besides a passive natural barrier for entrance of pathogens, the active role of the intestinal epithelium in pathogenesis of IBD.

Butyrate, the carbohydrate by-product by bacteria in the intestinal lumen, showed an efficacious protection in early UC lesions from the multicenter study in the United States, possibly by modulating the production of cytokines and/or chemokines (16). However, in vitro studies showed the controversial action of butyrate in intestinal epithelial cells, the up-regulation or down-regulation of the production of IL-8 can be observed in the different system $(3,5,17)$. IL-8, a member of C-X-C family of chemokines, attracts and activates neutrophils to the infection site and initiates the pathogenesis of IBD. The enhancement of IL- 8 by butyrate does not explain the potential protective effect from butyrate. Thus, the evaluation of butyrate in the treatment of IBD and the mechanism behind it have been an important matter of debate. Our experiments showed that butyrate could both decrease and increase the production of IL-8 in Pam3CSK4-triggered intestinal epithelia. At early time points, butyrate decreased the Pam3CSK4-triggered IL-8 production, but greatly enhanced it later. We hypothesized that this paradox was explicable by butyrate simultaneously enhancing IL-8 expression, but upregulating an inhibitor of NF- $\kappa \mathrm{B}$ in a time-limited manner.

Recently, many negative regulators of NF- $\kappa \mathrm{B}$ signaling pathway have been reported (18-20). An important member 
of the group of inhibitors is A20. The human A20 gene encodes a 790 -amino acid-containing protein of $90 \mathrm{kD}$. A20 is a cytoplasmic protein including a $\mathrm{N}$-terminal ovarian tumor (OTU) domain and a C-terminal seven zinc finger domain. A series of recent articles $(21,22)$ have demonstrated the role of A20 as a negative regulator in NF- $\kappa$ B pathways. For example, overexpression of A20 in HEK293 cells inhibited NF- $\kappa$ Bdependent gene expression induced by LPS or PGN. Similar effects were also observed in airway epithelium cell lines (AEC) HCI-H292. Furthermore, the full-length A20 inhibited the ability of TLR4 to activate the transcription factors, NF- $\kappa \mathrm{B}$ and AP-1, both of which induce the IL- 8 . The C-terminal zinc finger domain of A20 alone inhibits NF- $\kappa \mathrm{B}$, whereas the full-length A20 is necessary to inhibit the activation of AP-1. The TLR4 expression is very low (data not shown) in Caco-2 cells. We, therefore, chose TLR2 ligand Pam3CSK4 as a candidate to explore the mechanism of butyrate on transient decrease of IL-8. Our data showed that the expression of exogenous A20 (gain of A20 function) significantly decreased Pam3CSK4-triggered IL-8 production, whereas, knockdown of A20 significantly increased Pam3CSK4-triggered IL-8 production, indicating A20 is the negative regulator of NF- $\kappa \mathrm{B}$ pathway activated by Pam3CSK4 stimulation. Butyrate was found to up-regulate A20 and it also transiently down-regulated IL-8 expression in vitro. Furthermore, in human intestinal explants a protective effect of butyrate was observed.

Previous studies have sought to explain the mechanism underlying the inhibition of IL- 8 by butyrate. These include 1 ) a direct inhibition on the production of pro-inflammatory substances in the intestinal epithelium (12);2) an alteration on the expression of $\mathrm{I} \kappa \mathrm{B} \beta$ by butyrate to modulate the transcriptional activation of IL-8 gene (22); and 3) an increase in the expression of Heat shock protein 70 (Hsp 70), which acts as chaperones through protein-protein interaction and imparts an anti-inflammatory role by inhibiting IL-8 production in intestinal epithelium cells (5). Although this work does not exclude these mechanisms, the demonstration that butyrate upregulates A20 is likely to be an important factor, not previously recognized. Our studies do not exclude other inhibitors of NF- $\kappa \mathrm{B}$ from playing a role in the effects of butyrate. However, an examination of each inhibitor of NF- $\kappa \mathrm{B}$ was not the goal of our studies, as new regulators are still being discovered.

Our experiments showed that butyrate decreases the Pam3CSK4-stimulated IL-8 production at early time points, but enhances at later times. The enhancement of IL- 8 by butyrate at later time points suggests an undesirable effect of butyrate as treatment of IBD. It is one of the reasons to explain the limited use of butyrate enemas in IBD. However, butyrate has a wide variety of effects on the intestinal mucosa, and therefore other factors are likely to be important. Nevertheless, a greater understanding of the mechanisms of butyrate action will bring better insights in its use as a therapeutic agent, and into the biologic effects of foods that increase its luminal concentrations.

Acknowledgments. The authors thank Dr. Nanda Nanthakumar, director of the xenograft tissue core (PO1-DK33506), for his generous help in providing human fetal tissues.

\section{REFERENCES}

1. Dotan I, Mayer L 2002 Immunopathogenesis of inflammatory bowel disease. Curr Opin Gastroenterol 18:421-427

2. Fusunyan RD, Quinn JJ, Fujimoto M, MacDermott RP, Sanderson IR 1999 Butyrate switches the pattern of chemokine secretion by intestinal epithelial cells through histone acetylation. Mol Med 5:631-640

3. Fusunyan RD, Quinn JJ, Ohno Y, Macdermott RP, Sanderson IR 1998 Butyrate enhances interleukin(IL)- 8 secretion by intestinal epithelial cells in response to IL-1 $\beta$ and lipopolysaccharide. Pediatr Res 43:84-90

4. Huang N, Katz JP, Martin DR, Wu GD 1997 Inhibition of IL-8 gene expression in Caco-2 cells by compounds which induce histone hyperacetylation. Cytokine 9:27-36

5. Andoh A, Fujiyama Y, Hata K, Araki Y, Takaya H, Shimada M, Bamba T 1999 Counter-regulatory effect of sodium butyrate on tumor necrosis factor-alpha (TNF$\alpha$ )-induced complement C3 and factor B biosynthesis in human intestinal epithelial cells. Clin Exp Immunol 118:23-29

6. Wu GD, Huang N, Wen X, Keilbaugh SA, Yang H 1999 High-level expression of $\mathrm{IkB}-\beta$ in the surface epithelium of the colon: in vitro evidence for an immunomodulatory role. J Leukoc Biol 66:1049-1056

7. Ikuta T, Kan YW, Swerdlow PS, Faller DV, Perrine SP 1998 Alterations in protein-DNA interactions in the gamma-globin gene promoter in response to butyrate therapy. Blood 92:2924-2933

8. Kogut MH, Iqbal M, He H, Philbin V, Kaiser P, Smith A 2005 Expression and function of Toll-like receptors in chicken heterophils. Dev Comp Immunol 29:791807

9. Elewaut D, Didonato JA, Kim JM, Truong F, Eckmann L, Kagnoff MF 1999 NF-kappa B is a central regulator of the intestinal epithelial cell innate immune response induced by infection with enteroinvasive bacteria. J Immunol 163:14571466

10. Vidali G, Boffa LC, Bradbury EM, Allfrey VG 1978 Butyrate suppression of histone deacetylation leads to accumulation of multiacetylated forms of histones $\mathrm{H} 3$ and $\mathrm{H} 4$ and increased DNase I sensitivity of the associated DNA sequences. Proc Natl Acad Sci U S A 75:2239-2243

11. Gon Y, Asai Y, Hashimoto S, Mizumura K, Jibiki I, Machino T, Ra C, Horie T 2004 A20 inhibits Toll-like receptor 2- and 4-mediated interleukin 8 synthesis in airway epithelial cells. Am J Respir Cell Mol Biol 31:330-336

12. O'Reilly SM, Moynagh PN 2003 Regulation of Toll-like receptor 4 signalling by A20 zinc finger protein. Biochem Biophys Res Commun 303:586-593

13. Furuta GT, Dzus AL, Taylor CT, Colgan SP 2000 Parallel induction of epithelial surface-associated chemokine and proteoglycan by cellular hypoxia: implications for neutrophil activation. J Leukoc Biol 68:251-259

14. Kagnoff MF, Eckmann L 1997 Epithelial cells as sensors for microbial infection. J Clin Invest 100:6-10

15. Ohtsuka Y, Lee J, Stamm DS, Sanderson IR 2001 MIP-2 secreted by epithelial cells increases neutrophil and lymphocyte recruitment in the mouse intestine. Gut 49:526533

16. Breuer RI, Soergel KH, Lashner BA, Christ ML, Hanauer SB, Vanagunas A, Harig JM, Keshavarzian A, Robinson M, Sellin JH, Weinberg D, Vidican DE, Flemal KL, Rademaker AW 1997 Short chain fatty acid rectal irrigation for left-sided ulcerative colitis: a randomised, placebo controlled trial. Gut 40:485-491

17. Malago JJ, Koninkx JF, Tooten PC, van Liere EA, van Dijk JE 2005 Antiinflammatory properties of heat shock protein 70 and butyrate on Salmonellainduced interleukin-8 secretion in enterocyte-like Caco-2 cells. Clin Exp Immunol 141:62-71

18. Wald D, Qin J, Zhao Z, Qian Y, Naramura M, Tian L, Towne J, Sims JE, Stark GR, Li X 2003 SIGIRR, a negative regulator of Toll-like receptor-interleukin 1 receptor signaling. Nat Immunol 4:920-927

19. Gutierrez O, Pipaon C, Inohara N, Fontalba A, Ogura Y, Prosper F, Nunez G, Fernandez-Luna JL 2002 Induction of Nod2 in myelomonocytic and intestinal epithelial cells via nuclear factor- $\kappa$ B activation. J Biol Chem 277:41701-41705

20. Patel M, Xu D, Kewin P, Choo-Kang B, McSharry C, Thomson NC, Liew Fy 2005 Toll like receptor 2 agonist ameliorates established allergic airways inflammation by promoting Th1 response and not via regulatory $\mathrm{T}$ cells. J Immunol 174:7558-7563

21. Fukushima A, Yamaguchi T, Ishida W, Fukata K, Ueno H 2006 TLR2 agonist ameliorates murine experimental allergic conjunctivitis by inducing CD4 positive T-cell apoptosis rather than by affecting the Th1/Th2 balance. Biochem Biophys Res Commun 339:1048-1055

22. Fusunyan RD, Quinn JJ, Ohno Y, Macdermott RP, Sanderson IR 1998 Butyrate enhances interleukin(IL)- 8 secretion by intestinal epithelial cells in response to $\mathrm{IL}-1 \beta$ and lipopolysaccharide. Pediatr Res 43:84-90 